\title{
Preliminary Validation of the Wind Tunnel Based Flight Control System Evaluation Method
}

\author{
Min Huang ${ }^{1, a}$, Zhong-wei Wang ${ }^{2}$, Xing-Bao Yang ${ }^{1}$, Zhen-yun Guo ${ }^{2}$ and Yao-bin Niu ${ }^{2}$ \\ ${ }^{1}$ Dalian Naval Academy, Dalian, Liaoning, 116018, China \\ ${ }^{2}$ National University of Defense Technology, Changsha, Hunan, 410073, China
}

\begin{abstract}
As mathematical models of aircraft aerodynamics and rudder loadings always have to be built in the hardware-in-the-loop simulation, wind tunnel based flight control system (FCS) evaluation methods were proposed in order to test and evaluate the flight control systems under real aerodynamic and rudder loading environment. To validate the evaluation method, a wind tunnel based flight control system test was performed in a hypersonic wind tunnel facility. As the aircraft support rig in the wind tunnel is static, the aircraft angle of attack cannot be changed in this test. During the test, the elevator response, the lift force and the pitching moment were measured. By analysing the measured data, the elevator control performance of the pitch control system was determined, and the pitch angle was successfully predicted, but the open-loop pitch control performance was not determined. These results validate the feasibility of evaluating the elevator control performance and predicting the pitch angle of a FCS by the wind tunnel based FCS evaluation method.
\end{abstract}

\section{Introduction}

Traditionally, after a flight control system is evaluated and confirmed by the hardware-in-the-loop simulation (HILS), it should then be tested and evaluated by the flight tests. However, the aircraft aerodynamics and rudder loadings in the HILS have to be mathematical models [1] which will make the HILS always have a distance away from the real flight tests. In order to replace these mathematical models with real factors, the aircraft installed with FCS hardware is directly put inside the wind tunnel which can make the wind tunnel airflow act on the aircraft body as in real flights. Then, the aircraft can rotate in the wind tunnel under the action of the aircraft flight control system [2]. Obviously, this kind of wind tunnel based FCS test is more realistic and closer to the flight tests than the HILS, especially in the case of modelling unsteady or nonlinear motions. If such a test is used to evaluate the FCS after HILS and before flight tests, more realistic FCS evaluation results will be obtained, and some FCS errors that can hardly be exposed in HILS, such as the inefficiency of the FCS under unsteady motions (in 2009, the hypersonic vehicle HTV-2 experienced a flight failure caused by the inefficiency of the FCS under the coupled pitch-roll motion [3]), can be detected by the wind tunnel based FCS tests. Therefore, the wind tunnel based FCS tests can make up the deficiency of HILS by putting the aircraft inside the wind tunnel, and reduce the risk of flight tests by exposing and solving some flight problems on the ground.

\footnotetext{
${ }^{\text {a }}$ Corresponding author : hmjtxy@163.com
} 
Due to the advantages of wind tunnel tests with control, many departments were developing the wind tunnel test techniques with control [4] in an attempt to predict flight performances and evaluate the flight control laws in wind tunnels. The Arnold Engineering Development Center (AEDC) [5] built an 8-wire suspended 2-DOF (degrees-of-freedom) wind tunnel test system with control and the Physical Sciences Incorporation (PSI) [6] in USA built an 6-wire suspended 3-DOF (pitch, yaw and roll) wind tunnel test system with control to reappear and predict the coupling motions that may occur in the real flights. The University of Cambridge [7] and the University of Bristol [8] in UK, the SibNIA [9] in Russia, the Royal Institute of Technology [10] in Sweden, the French-German Research Institute of Saint-Louis [11] in France, the China Aerodynamics Research and Development Center (CARDC) [12-13] in China all had built wind tunnel test systems with control to evaluate the flight control laws. However, up to now, the wind tunnel tests for evaluating the flight control systems have not been developed yet. Therefore, the wind tunnel tests for evaluating the flight control systems should be researched.

According to the above demand for the wind tunnel based FCS evaluation methods, the wind tunnel based FCS evaluation method for FCS was proposed in references [14-15]. To preliminarily validate the evaluation method, a wind tunnel based flight control system test was performed in a hypersonic wind tunnel, and the test data was analyzed to judge whether the FCS performances can be determined by the evaluation method. If some or the whole performances of the pitch control system are determined, such as the elevator control performance and the open-loop or the closed-loop pitch control performances, the evaluation method can be partly or fully validated.

\section{The wind tunnel based flight control system evaluation method}

Before introducing the wind tunnel based flight control system test, the wind tunnel based flight control system evaluation method proposed in references [14-15] is generally introduced here.

A classic unmanned pitch control system (shown in Figure 1) was taken as the evaluation object in the wind tunnel based flight control system evaluation method. The followings are the details towards this method.

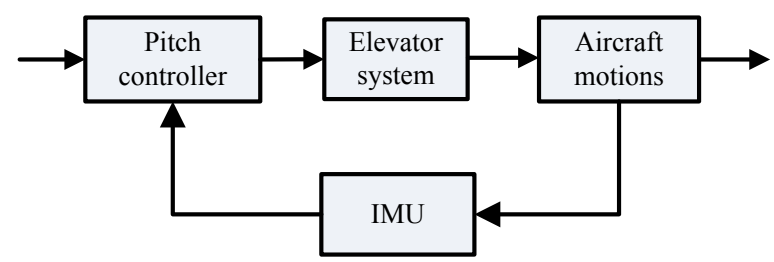

Figure 1. The structure of an unmanned pitch control system.

Firstly, the test system scheme of this evaluation method is shown in Figure 2. This system is mainly consisted of four parts: a wind tunnel facility, a full-scaled aircraft test article with FCS hardware installed inside, a test article support rig with an aerodynamic balance installed on it, a realtime data acquisition and control system (short as RDAS). The wind tunnel facility generates the same airflow as that of the real flight; i.e. the airflow velocity, density, viscosity, and static pressure in the wind tunnel are the same as that in real flight. The full-scaled aircraft test article which is the same as that in real flight is put inside the wind tunnel. In this way, the aerodynamic/motion/control in this wind tunnel test can be consistent with the real flight. The test article support rig is used for driving the test article to rotate according to the computed angle of attack command so as to provide an equivalent attitude response. The RDAS is used for collecting data measured in the wind tunnel, processing data and transmitting processed data to the support rig and the controller in the wind tunnel.

Secondly, the working principle of the above system for testing the FCS is introduced here. Before a wind tunnel test, a pitch angle command should be preprogramed into the controller. At the beginning of a wind tunnel test, the RDAS should send an airflow command to the wind tunnel facility so as to generate a desired airflow. During the test, the IMU measures the angle of attack $\alpha$ 
and the aerodynamic balance measures the lift force $L$, which are then sent to the RDAS. With the measured $\alpha$ and $L$, the RDAS computes the pitch angle feedback with equation (1) which is then sent to the controller. The controller combines the pitch angle command and the pitch angle feedback, and generates an elevator angle command for the servo which will then drive the elevator to deflect. Once after the elevator deflects, the balance measures the pitching moment $M_{z}$ which is then sent to the RDAS. The RDAS use the measured $M_{z}$ to compute a new angle of attack command for the support rig with equation (2). Then the support rig will drive the aircraft test article to the new angle of attack. And the RDAS will compute a new pitch angle feedback for the controller until the feedback pitch angle reaches to the pitch angle command.

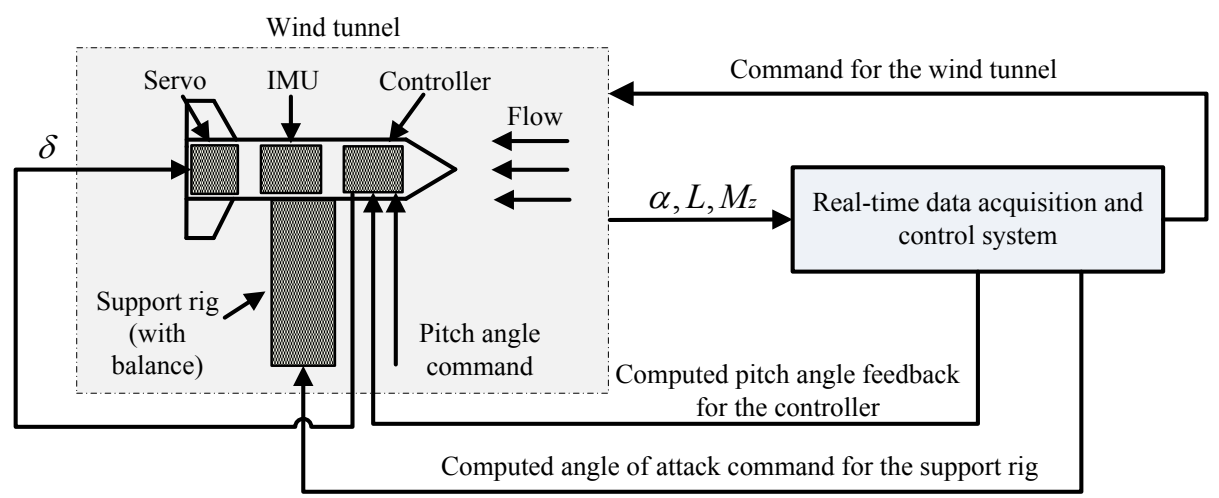

Figure 2. The wind tunnel based FCS test system scheme for the pitch control system.

$$
\begin{gathered}
\dot{\theta}=\frac{T \sin \alpha+L}{m V}-\frac{g \cos \theta}{V} \\
\varphi=\theta+\alpha \\
\ddot{\alpha}=M_{z} / I_{z}
\end{gathered}
$$

In the above equations (1 2), $I_{z}$ is the pitching inertia moment, $\theta$ is the longitudinal velocity angle, $T$ is the thrust (which is set as 0 in this paper), $m$ is the aircraft mass, $g$ is the local acceleration of gravity, $V$ is the aircraft speed, $\varphi$ is the pitch angle.

Then, according to the working principle of the test system introduced above, closed-loop or openloop pitch control tests of the pitch control system can be performed. With the collected data from these tests, the pitch control performances can be determined.

To validate the above evaluation method, wind tunnel based FCS tests according to the evaluation method should be performed. In this paper, a hypersonic wind tunnel facility is used for the validation.

\section{A hypersonic wind tunnel test system for validating the evaluation method}

The hypersonic wind tunnel test system for validating the evaluation method includes a hypersonic wind tunnel facility, a full-scaled hypersonic aircraft test article with FCS hardware installed inside, a 5-degree-of-freedom abdominal support rig, a water cooling equipment installed on the support rig for cooling the rig, an aerodynamic balance installed on the rig, and a RDAS. The support rig can move on the angle of attack degree-of-freedom, the angle of sideslip degree-of-freedom, and three translational degree-of-freedoms as shown in Figure 3. As the rig is static during the tests, the rig should be adjusted to and fixed at an appropriate position before a wind tunnel test. The RDAS includes the aircraft data acquisition system (short as ADAS), the wind tunnel data acquisition and 
control system (short as WTDAS). The ADAS mainly collects the test data from the aircraft test article, such as the angle of attack and the elevator angle. The WTDAS mainly collects the test data from the wind tunnel test devices, such as the aerodynamic forces and moments, the airflow data.

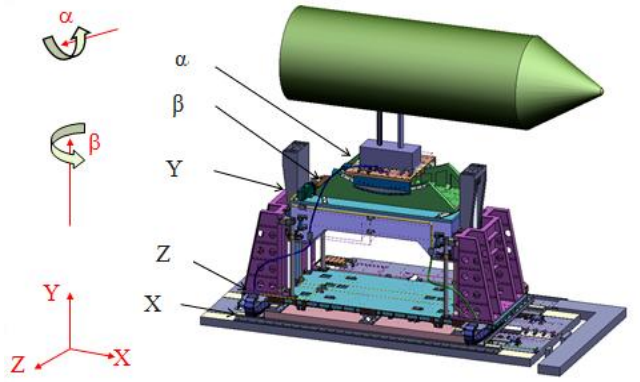

Figure 3. Sketch map of the 5-DOFs abdominal support rig in the hypersonic wind tunnel.

As the support rig cannot move during the tests, dynamic aircraft wind tunnel tests cannot be performed. Therefore, only the static open-loop pitch control test was performed to preliminarily validate the evaluation method. The open-loop pitch control test scheme is introduced as follows, including its test loop, pitch control law and test operation procedure.

\section{The open-loop pitch control test based on the hypersonic wind tunnel system}

\subsection{The pitch control test loop}

The open-loop pitch control test loop is shown in Figure 4. Before the test, the aircraft is fixed at a specific angle of attack $\alpha_{i}$ by the support rig which is set as 0 degree. And a control command $\alpha_{c}$ is preprogramed into the controller. The $\alpha_{c}$ is a sinusoidal input and equates to $2 \sin \left(2 \pi t / T_{0}\right)$. The time period $T_{0}$ is set as 3 seconds. As the support rig and the aircraft test article are static during the test, the RDAS just collects the elevator angle , $L$, and $M_{z}$, and doesn't feedback the computed data to the controller and the support rig.

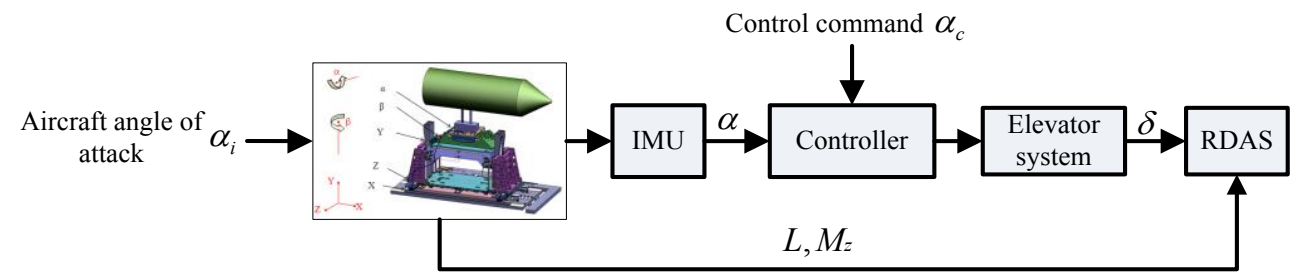

Figure 4. Sketch map of the open-loop pitch control test loop based on the hypersonic wind tunnel test system.

The collected elevator response is used to analyse and determine the elevator control performance of the pitch control system. And the collected $L$ and $M_{z}$ are used to predict the pitch angle with equations (1) (2) and analyse the open-loop pitch control performance.

\subsection{Pitch control law}




\subsubsection{The control law}

The classic PID control method is used for pitch controller. The control law of the pitch controller [16] is:

$$
\left\{\begin{array}{c}
e(t)=\alpha_{c}(t)-\alpha_{i} \\
\delta(t)=K_{p} e(t)+K_{i} \int_{0}^{t} e(\tau) d \tau+K_{d} \frac{d e(t)}{d t}
\end{array}\right.
$$

Thereinto, the PID control parameters are set as: $K_{p}=2, K_{i}=0.2, K_{d}=1$.

\subsubsection{Simulation validation of the control law}

As the hypersonic wind tunnel test requires that the elevator angle should be less than 10 degree, the PID control law and the control parameters were validated by the mathematical simulation so as to ensure that the largest elevator angle is less than $10^{\circ}$. By inputting the values of $\alpha_{i}$ and $\alpha_{c}$ into the equation (3), the elevator response was computed. The computed largest elevator angle $\delta_{\max }$ $=5.63^{\circ}<10^{\circ}$, which validate the PID control law and parameters.

\subsection{Test operation procedure}

The open-loop pitch control test was performed according to the following procedure:

(1) Adjust the position of the support rig and fix the aircraft test article at $0^{\circ}$ angle of attack;

(2) Turn on the aircraft, and return the elevator angle to $0^{\circ}$;

(3) Turn on the wind tunnel, and let it generate airflow with the speed of $5 \mathrm{Ma}$;

(4) After a while when the airflow gets steady, the elevator is controlled to deflect;

(5) After 6 seconds, the elevator stops deflecting;

(6) Turn off the wind tunnel, and finish the test.

\section{Analysis of the test data and determination of the pitch control system performances}

Based on the hypersonic wind tunnel test system in Section 3, and according to the test scheme in Section 4, the open-loop pitch control test was performed. The elevator response and the aerodynamic responses were obtained. The elevator response was used to determine the elevator control performance. If the elevator control performance is successfully determined, the feasibility of the wind tunnel based FCS evaluation method for evaluating the elevator control performance of a FCS can be validated. The aerodynamic responses were used to predict the pitch angle response and determine the open-loop pitch control performance. If the open-loop pitch control performance is successfully determined, the feasibility of the wind tunnel based FCS evaluation method for evaluating the open-loop pitch control performance of a FCS can be validated.

\subsection{Analysis of the elevator response data and determination of the elevator control performance}

$\delta$ The ADAS collected elevator response is shown in Figure 5. The collected elevator response curve is almost identical to the elevator command curve. But some error exists. 


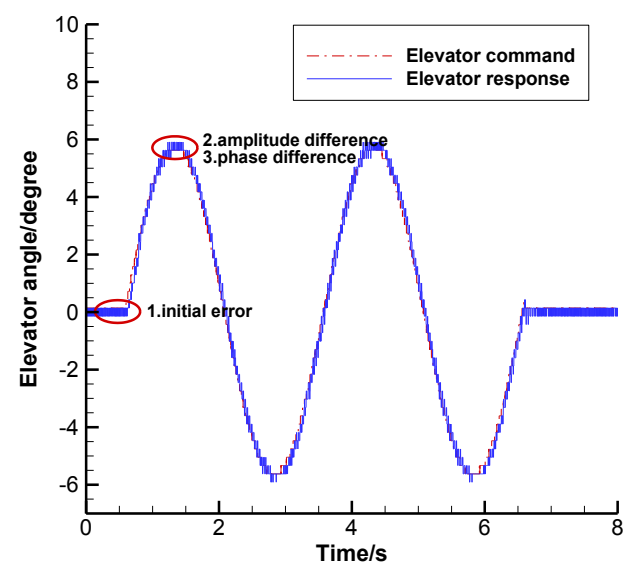

Figure 5. ADAS collected elevator response.

Firstly, before the elevator deflects, an initial error exists, which is mainly caused by the systemic errors, such as the elevator structure oscillation, the measuring error or the collecting error. The initial error is named as $\Delta_{i}$. By subtracting the initial elevator command from the initial elevator response, the $\Delta_{i}$ can be obtained:

$$
\Delta_{i}=0.432^{\circ}-0.144^{\circ}=0.288^{\circ}
$$

Actually, such a noise error also always exists in the collected elevator response. Here, it is supposed that the collected elevator angles at all the time have the same noise error $\Delta_{i}$. Therefore, when comparing the elevator command with the elevator response, the $\Delta_{i}$ should be subtracted from the elevator response.

Secondly, by comparing the elevator response and the elevator command, the amplitude difference $A_{\delta}$ and the phase difference $T_{\delta}$ can be obtained to determine the elevator control performance. By reading data from Figure 5, the maximum angle of the elevator response is $5.911^{\circ}$, the maximum angle of the elevator command is $5.63^{\circ}$, and then the amplitude difference is:

$$
A_{\delta}=\left|5.911^{\circ}-5.63^{\circ}\right| / 5.63^{\circ} \times 100 \%=0.124 \%
$$

The time for the elevator response and the elevator command to reach to their maximum angles were chosen to compute their phase difference. The time for the elevator response to reach to its maximum angle $5.911^{\circ}$ is $1.141 \mathrm{~s}$, the time for the elevator command to reach to its maximum angle $5.63^{\circ}$ is $1.204 \mathrm{~s}$, and then the phase difference is:

$$
T_{\delta}=|1.204 \mathrm{~s}-1.141 \mathrm{~s}|=0.063 \mathrm{~s}
$$

As the elevator control performance indexes $A_{\delta}$ and $T_{\delta}$ are not 0 , the elevator response will have a difference with the elevator command. This elevator angle tracking error will lead to the attitude angle tracking error, which will then affect the attitude control performances. Specifically, the attitude control rapidity and accuracy will be reduced. But as the indexes $A_{\delta}$ and $T_{\delta}$ are very small and are quite allowable in the control system, it can be judged that the elevator response tracks the elevator command very well, which demonstrates that the pitch control system has a good elevator control performance. Through this successful determination of the elevator control performance, the 
feasibility of the wind tunnel based FCS evaluation method for evaluating the elevator control performance of a FCS was validated.

\subsection{Analysis of the aerodynamic data and determination of the open-loop pitch control performance}

\subsubsection{Analysis of the aerodynamic data}

The WTDAS collected aerodynamic response data including the lift force $L$ and the pitching moment $M_{z}$ is shown in Figure 6.

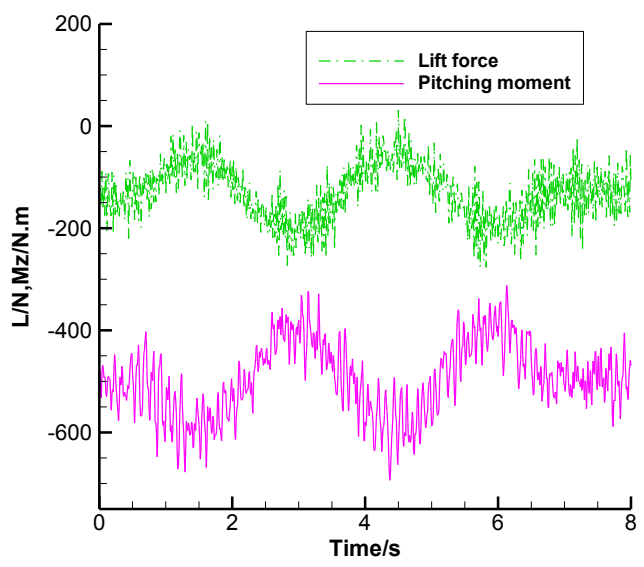

Figure 6. WTDAS collected aerodynamic responses.

The aerodynamic response data was analyzed as follows:

(1) The collected lift force response $L$ curve in Figure 6 is just opposite to that of the elevator response in Figure 5, and the trend of $M_{z}$ curve is correspondent to that of the elevator response. This shows that: within the deflection range of elevator angle, as the elevator angle changes, the lift force $L$ will change negatively with the elevator angle and the pitching moment $M_{z}$ will change positively with the elevator angle.

(2) As the collected lift force and pitching moment in Figure 6 contain obvious noise, data filtering is needed here. A low pass filter based on the FFT was used to filter these two data. The filtered aerodynamic response data without noise was shown in Figure 7.

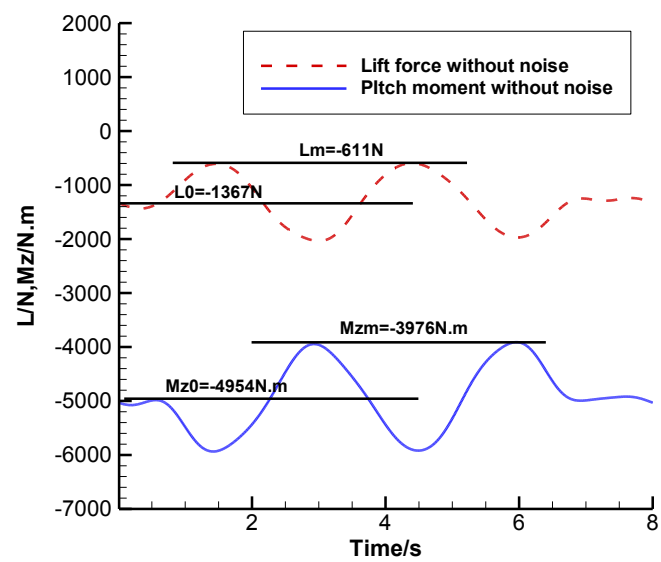

Figure 7. Aerodynamic responses without noise. 
(3) For the convenience of computing the pitch angle response, the equations for computing the aerodynamic response should be constructed. For this purpose, firstly, the mean lines at the medium position and the maximum position of the aerodynamic responses were respectively drawn and their aerodynamic values were marked in Fig 8. Secondly, the lift force magnitude $A_{L}$ and the pitching moment magnitude $A_{M z}$ were computed: $A_{L}=L m-L_{0}=75.6 \mathrm{~N}, A_{M z}=M z m-M z_{0}=97.8 \mathrm{~N}$.m. Thirdly, according to their initial values, magnitudes and time period $T_{0}$ (which is $3 \mathrm{~s}$ ), their standard aerodynamic response equations were constructed:

$$
\begin{gathered}
L=-136.7+75.6 \sin \left(2 \pi t / T_{0}\right) \\
M_{z}=-495.4-97.8 \sin \left(2 \pi t / T_{0}\right)
\end{gathered}
$$

\subsubsection{Prediction and analysis of the pitch angle response}

By inputting the above aerodynamic equations (4) (5) into the equations (1) (2), the angle of attack and the pitch angle response was computed as shown in Figure 8.

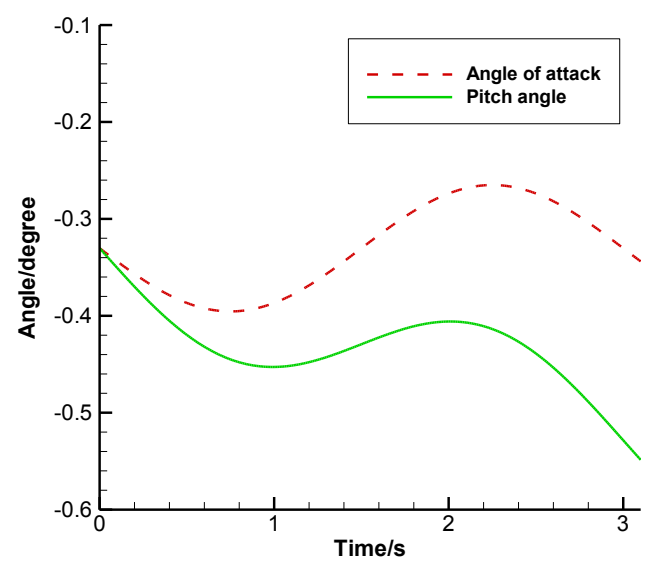

Figure 8. Predicted angle of attack and pitch angle responses.

From the curve, it can be clearly seen that the maximum pitch angle is far less than the maximum control command which is 2 degree, but the trend is consistent with the control command. As for why the pitch angle response is this small, the reason is that the aircraft angle of attack in this wind tunnel test is always 0 and the pitching moment is correspondingly very small which leads to small pitch angle response.

\subsubsection{Determination of the open-loop pitch control performance}

Although through the above test and data processing, the pitch angle was successfully predicted, it cannot be used to determine the open-loop pitch control performance. This is because the aircraft angle of attack in this test didn't change and the predicted pitch angle is not dynamically real and cannot be compared with the control command. Therefore, the current static support rig should be improved to a dynamic rig so as to perform dynamic wind tunnel based FCS tests, obtain the dynamic lift force and pitching moment and then the dynamically real pitch angle can be predicted. In this way, the open-loop pitch control performance and even the closed-loop pitch control performance can be determined.

To sum up the above analyses, the elevator control performance of the pitch control system was successfully determined, and the pitch angle response was successfully predicted. These validate the 
feasibility of part of the wind tunnel based FCS evaluation method, i.e. the feasibility of evaluating the elevator control performance and predicting the pitch angle of a FCS by this method. Although the open-loop pitch control performance was not determined, the test and data analysis work can lay foundations for future performing of more integrated tests and validating more of the evaluation method.

\section{Conclusions}

The wind tunnel based flight control system evaluation method can evaluate the FCS under real aerodynamics and rudder loadings, which make this method more realistic than the traditional hardware-in-the-loop simulation evaluation method. With a hypersonic wind tunnel test system, a static open-loop pitch control test of the pitch control system was performed and used to validate this method in this paper.

Through this test, the elevator response and the aerodynamic responses were collected. By analysing the test data, the elevator control performance of the pitch control system was successfully determined, and the pitch angle response was successfully predicted, which validate the feasibility of evaluating the elevator control performance by the wind tunnel based FCS evaluation method. As the support rig and the aircraft test article cannot move during the wind tunnel tests, the predicted pitch angle is not dynamically real and the open-loop pitch control performance was not determined. But the test and data analysis work in this paper can lay foundations for future performing of the dynamic open-loop or even closed-loop pitch control tests, successful determining the pitch control performances and fully validating the evaluation method.

\section{References}

1. Shan J Y, Meng X Y, Ding Y. Hardware in the loop simulation. Beijing: National Defence Industry Press, 2008. (in Chinese)

2. Li Z F. Special wind tunnel test techniques. Beijing: Aviation Industry Press, 2010. (in Chinese)

3. Li H F. Guidance and control techniques of hypersonic aircrafts. Beijing: Chinese aerospace press, 2012. (in Chinese)

4. Huang M, Wang Z W. A Review of Wind Tunnel Based Virtual Flight Testing Techniques for Evaluation of Flight Control Systems. International journal of aerospace engineering, 2015(1), 122.

5. Lawrence C, Mills B. Status update of the AEDC virtual flight testing development program. 40th AIAA Aerospace Sciences Meeting \&Exhibit, AIAA 2002-0168, Reno, USA, 14-17 January, 2002.

6. Magill J C, Cataldi P, Morency J R, Hammer D X, Burgess R, Jeter E. Demonstration of a wire suspension for wind-tunnel virtual flight testing. Journal of Spacecraft and Rockets, 46(3): 624633, 2009.

7. Papageorgiou G and Glover K. Two-degree-of-freedom control of an actively controlled windtunnel model. Journal of Guidance Control and Dynamics, 25(3): 510-516, 2002.

8. Richardson T S, Dubs A, Lowenberg M H, and Jones C D. Wind-tunnel testing of a dynamic state-feedback gain scheduled control system. Proceedings of the AIAA Guidance, Navigation and Control Conference and Exhibition, AIAA 2005-5976, San Francisco, California, USA, 1518 August, 2005.

9. Sohi NP. Modeling of spin modes of supersonic aircraft in horizontal wind tunnel. Proceedings of the 24th International Congress of the Aeronautical Sciences, Yokohama, Japan, September, 2004.

10. Stenfelt G, Ringertz U. Yaw control of a tailless aircraft configuration. Journal of aircraft, 47(5): 1807-1810, 2010.

11. Strub G, Theodoulis S, Gassmann V, Dobre S. Pitch Axis Control for a Guided Projectile in a Wind Tunnel-based Hardware-In-the-Loop Setup. AIAA Modeling and Simulation Technologies Conference, AIAA 2015-0153, 5-9 January, 2015. 
12. Zhao Z L, Wu J Q, Li H, Zhou W Q, Mao D Y, Yang H Y. Investigation of virtual flight testing technique based on $2.4 \mathrm{~m}$ transonic wind tunnel. Acta Aeronautica et Astronautica Sinica, 37(2): 504-512, 2016. (in Chinese)

13. Nie B W, Zhu M H, Guo L L, Wen Y C, Jiang M. Key technique and design scheme of the wind tunnel virtual flight system. In: 2012 Chinese Guidance, Navigation and Control Conference, Beijing, China, 10-12 August, 2012.

14. Huang M, Wang Z W, Guo Z Y, Niu Y B. Design of the wind tunnel based virtual flight testing evaluation method for flight control systems. Proc IMechE Part G: Journal of Aerospace Engineering, 0(0): 1-13, 2016.

15. Huang M, Wang Z W, Guo Z Y, Zeng Q H, Niu Y B. Wind tunnel hardware-in-the-loop simulation techniques for flight control system evaluation. 2017 2nd International Conference on Control, Automation and Artificial Intelligence, 2017.

16. Huang Y R, Qu L G. Parameter adjusting and realizing of PID controllers. Beijing: Science press, 2010. (in Chinese) 